\title{
EL PUERTO FENICIO-PÚNICO DE GADIR: UNA NUEVA VISIÓN DESDE LA GEOARQUEOLOGÍA URBANA DE CÁDIZ
}

\section{THE PHOENICIAN-PUNIC HARBOUR OF GADIR. A NEW PERSPECTIVE FROM THE URBAN GEOARCHAEOLOGY OF CÁDIZ}

\author{
por \\ OSWALDO ARTEAGA y \\ ANNA-MARÍA ROOS
}

A don Manuel Pellicer con el afecto de los autores.

\begin{abstract}
RESUMEN En atención a las dudas suscitadas acerca de la ubicación del Puerto Fenicio de Gadir damos a conocer los resultados geoarqueológicos que en la primavera del año 2001 han permitido su descubrimiento en el subsuelo del casco antiguo de Cádiz. Se confirma la continuidad histórica de la ciudad portuaria de Gadir con la 'Polis Púnica Gaditana' y con la Gades romana.
\end{abstract}

\begin{abstract}
In order to remove the doubts caused about the location of Gadir Phoenician Seaport, we make known the Geoarchaeological results of the perforations carried out in the campaingn in spring 2001. They have allowed us to define the existence of an 'Inside Seaport' that we can identify with the characteristics of the 'Gadir' Phoenician Seaport in the Historic Centre of Cadiz. Consequently, the Geoarchaeological sequence confirms an uninterrupted historical continuity among the Phoenician 'Gadir', the Punic Polis and the Roman 'Gades'.
\end{abstract}

Palabras claves Geoarqueología. Gadir. Gades.

Key words Geoarchaeology. Gadir. Gades.

En recientes trabajos publicados venimos dando cuenta de las Investigaciones Geoarqueológicas que en colaboración con el prof. Dr. Horst D. Schulz y contando con la participación de un competente equipo interdisciplinario de las Universidades de Sevilla (Arqueología) y Bremen (Geología) hemos continuado llevando a cabo, tomando el relevo de los trabajos iniciados hace veinte años en el seno científico del Instituto Arqueológico Alemán de Madrid (Schulz 1983; Arteaga et alii 1985; 1988). 
Estas investigaciones geoarqueológicas, a partir de 1992 (Arteaga y Roos 1992; 1995; Schulz et alii 1992; 1995; Arteaga, Schulz y Roos 1995) han seguido teniendo por objeto el estudio de las incidencias socio-históricas que siendo analizadas como 'causas antrópicas' durante el Holoceno (Arteaga y Hoffmann 1999) vienen coadyuvando a los cambios observados en el paisaje litoral de Andalucía (Arteaga y Schulz 2000) y por lo tanto en la variación de las antiguas líneas costeras, en este ámbito atlántico-mediterráneo de la Península Ibérica, hasta nuestros días.

Con la gustosa intención de recordar el interés científico mostrado por el prof. Dr. Manuel Pellicer Catalán en relación con esta misma problemática, siendo como bien sabemos uno de los autores pioneros en abordar una propuesta de ubicación costera relativa a la situación de los primeros asentamientos fenicios en Andalucía (Pellicer 1962; Schubart, Niemeyer y Pellicer 1969) y más concretamente con motivo de la localización del establecimiento conocido ahora como Cerro del Prado, cerca de Algeciras (Pellicer, Menanteau y Rouillard 1977) nosotros queremos rememorar aquella feliz iniciativa en la oportunidad de dedicarle esta breve reseña geoarqueológica sobre la ubicación del Puerto de Gadir: sumándonos con afecto al merecido homenaje que ahora se le rinde, en reconocimiento de su larga trayectoria profesional como arqueólogo de campo, y como docente universitario.

\section{PERSPECTIVAS DE LA GEOARQUEOLOGÍA HOLOCENA EN EL LITORAL ATLÁNTICO- MEDITERRÁNEO DE ANDALUCÍA: EL REFERENTE PORTUARIO DE LOS FENICIOS}

Las aplicaciones analíticas que actualmente hacemos respecto de la Geoarqueología del Holoceno obedecen a una desiderata teórica y metodológica bastante poco desarrollada en Andalucía hasta comienzos de los años ochenta (Schulz 1983; Arteaga et alii 1985; 1988).

Esta renovación teórica (Arteaga y Hoffmann 1999) y metodológica (Arteaga y Schulz 2000) conoce sobre todo unos antecedentes que se fundamentaron en la necesidad de solventar una incógnita más que nada planteada en relación con la ubicación que mostraban 'tierra adentro', respecto de la línea costera actual (Schubart, Niemeyer y Pellicer 1969; Arribas y Arteaga 1975; Pellicer, Menanteau y Rouillard 1977), algunos asentamientos portuarios que según las fuentes escritas greco-latinas habrían fundado los navegantes fenicios en el litoral atlántico-mediterráneo de Andalucía. Es decir, en las inmediaciones de los actuales sitios de Cádiz, Málaga, Almuñécar y Adra. Y asimismo, por lo tanto, en relación con otros muchos asentamientos secundarios, que no siendo 'nominados' por dichas fuentes como unos establecimientos principales, la arqueología de campo venía poniendo igualmente en evidencia: como sería el caso del mencionado Cerro del Prado (Pellicer, Menanteau y Rouillard 1977), y como ya lo mostraban también las excavaciones practicadas después del famoso descubrimiento de la Necrópolis Laurita (Pellicer 1962) en las factorías fenicias de Toscanos (Schubart, Niemeyer y Pellicer 1969) y del Guadalhorce (Arribas y Arteaga 1975).

En definitiva, se trataba de un problema geomorfológico de difícil solución dependiendo como estaba de la aplicación de unos medios puramente arqueológicos, sin el concurso de la Geología, de la Geografía Física, y de otras disciplinas afines. Ya que resultaba evidente que después de los tiempos protohistóricos la línea de la costa había estadó cambiando de lugar hasta alcanzar su actual situación, quedando por lo mismo los antiguos puertos fenicios ahora retirados varios kilómetros hacia el hinterland (Schubart, Niemeyer y Pellicer 1969; Arribas y Arteaga 1975; Pellicer, Menanteau y Rouillard 1977).

En un principio, estas preocupaciones geomorfológicas pasaron por el reconocimiento de una carencia interdisciplinaria, como aquella que algunos geólogos, geógrafos y arqueólogos habían señalado como una acuciante necesidad científica desde varias décadas anteriores (Gavala 1927; 1959; Pemán 1941; García Bellido 1945; Menanteau 1982). 
En este caldo de cultivo, los intentos corográficos que hasta los años setenta se sumaban iban procurando 'superar' el presentismo de los mapas actuales, corrientemente utilizados para la localización puntual de los llamados 'yacimientos' fenicios, dibujando solamente de una manera tentativa las supuestas líneas de la costa a tenor de las curvas de nivel que aquellos autores suponían entonces como las más probables, teniendo en cuenta sobre todo las alturas relativas a la cota topográfica de los diez metros sobre el nivel del mar.

Mediante el repetido recurso de la aplicación de esta 'técnica' basada en dibujos topográficos calcados en 'papel cebolla', para salir del paso se continuaron copiando y se publicaron unas ingeniosas representaciones cartográficas, supuestamente costeras. Resultando hasta nuestros días manidamente emulativas de aquellas simulaciones virtuales que tomando por guía los mapas geológicos de autores como Juan Gavala y Laborde (1959), buscaron aproximarse con unos propósitos meramente ilustrativos a la posible mostración física del Archipiélago de Cádiz; para ensayar aquí mismo incluso distintas ubicaciones topográficas, intentando 'mejorar' las que otros arqueólogos pioneros habían propuesto acerca de los asentamientos fenicios de Gadir (Pemán 1941; García Bellido 1945).

Por esto último cabe retener que antes de poder proponer por nuestra parte las aplicaciones geoarqueológicas que hemos experimentado desde hace veinte años (Arteaga et alii 1985) nosotros mismos tuvimos que estar abiertos a la admisión de una crítica metodológica acerca de aquellas prácticas descriptivas y para nada explicativas. Y que siendo similares a las reproducidas para Cádiz (diversas alternativas reseñadas en Aubet 1994: fig. 63) y a las que continuaron apareciendo referidas a diversos sitios portuarios atlánticosmediterráneos (Gras, Rouillard y Teixidor 1989: 257-262) tendríamos mutatis mutandis que abandonar al paso en que tomábamos conciencia de la dificultad que entrañaba la ubicación portuaria de aquellos asentamientos fenicios que estudiábamos en las costas mediterráneas de Andalucía hacia principios de los años ochenta (Arribas y Arteaga 1975: fig. 2; Schubart 1982).

No obstante, hemos de añadir que los fructíferos debates paleogeográficos que pudimos asimilar quienes como estudiantes participábamos en las investigaciones iniciadas por los profesores Schubart, Niemeyer y Pellicer (1969), en los asentamientos fenicios de la actual desembocadura del río de Vélez cerca de Torre del Mar y que entonces eran continuadas por el Instituto Arqueológico Alemán de Madrid, teniendo siempre en cuenta los antecedentes mencionados, fueron los mismos debates que a partir del año 1982 (una síntesis en: Arteaga y Schulz 2000) nos posibilitaron replantear como también estaban proponiendo otros arqueólogos (Schüle 1986: 208-214) el estudio de las líneas costeras conocidas por los fenicios, cuando fundaron los primeros asentamientos coloniales en el ámbito atlántico-mediterráneo de Andalucía. Siendo así desde el fuero interno de dicha experimentación como partiendo de una misma expectativa de representación cartográfica a todas luces 'tradicional' (Arribas y Arteaga 1975: fig. 2), por la iniciativa honrosa de otro de sus usuarios (Schubart 1982) en los debates de la Axarquía malagueña se potenciarían primero los ensayos de la praxis metodológica encaminada a la contrastación de un nuevo derrotero interdisciplinario, y en segundo término a la proyección de un futuro programa de investigación, abordando de este modo la extensión atlántico-mediterránea de las desideratas planteadas (Arteaga et alii 1985).

Los primeros ensayos metodológicos en colaboración con la Universidad de Kiel (Schulz 1983; Schulz, Jordt y Weber 1988) quedaron concretados en 1984, mediante la excavación sistemática realizada en relación con la ubicación del Puerto Fenicio de Toscanos (Arteaga 1988; Schulz 1988). Y la contrastación analítica derivada de aquella experiencia científica (Arteaga et alii 1985) potenciada una vez más por parte de la dirección del Instituto Arqueológico Alemán de Madrid, contando entonces con las competencias geológicas de la Universidad de Bremen, y con el apoyo institucional de la Dirección General de Bienes Culturales de la Consejería de Cultura de la Junta de Andalucía (Arteaga et alii 1985) quedaría plasmada en la realización del llamado 'Proyecto de la Costa', sobre el cual contamos con algunas síntesis preliminares (Hoffmann 1988; Arteaga y Hoffmann 1999). 
En suma, podemos apuntar que una vez completadas las primeras actuaciones 'mediterráneas' previstas, se comenzaron las investigaciones geoarqueológicas en la parte 'atlántica' de Andalucía, mediante unas perforaciones practicadas en la zona del Bajo Guadiana (Schubart et alii 1988).

Desde el año 1992 en adelante, tomando el relevo del cometido propuesto, una nueva colaboración establecida entre las Universidades de Sevilla (España) y de Bremen (Alemania) asumiría la aplicación de la citada metodología experimentada a lo largo de diez años, ahora en el estudio de dos zonas de especial interés:

a) La referida al Proyecto Geoarqueológico de las Marismas del río Guadalquivir (Schulz et alii 1992; 1995; Arteaga y Roos 1992; 1995; Arteaga, Schulz y Roos 1995).

b) La concerniente al Proyecto Geoarqueológico de la Bahía de Cádiz (Schulz et alii, en prep.; Arteaga y Roos, en prep;; Arteaga et alii 2001).

Puesto que estas actividades apenas iniciadas se encuentran todavía inacabadas, cabe también adelantar que en estos momentos pueden enumerarse en concreto cuáles habrán de ser las actuaciones programadas para abarcar por completo la Geoarqueología Holocena del Litoral de Andalucía, y para conectar sus resultados comparativos con un ambicioso programa de Geoarqueología Atlántica, incluyendo diversas actividades contrastadas en diferentes estuarios de Europa Occidental y de América. Para llevar a cabo esta magna empresa, en el año 2001 se acaba de concertar un nuevo convenio de investigación coordinado por Oswaldo Arteaga desde la Arqueología (Univ. Sevilla, España) y coodirigido por Horst D. Schulz para la Geología (Univ. Bremen, Alemania). Incorporándose también de ahora en adelante Loïc Menanteau para las competencias que corresponden a la Geografía (Univ. Nantes, Francia). Las actuaciones previstas, entre los años 2003 y 2010, comprenden de momento los siguientes programas en particular:

a) Culminación de la Geoarqueología Holocena del Bajo Guadalquivir.

b) Culminación de la Geoarqueología Holocena de la Bahía de Cádiz.

c) Culminación de la Geoarqueología Holocena de la Costa Onubense: en los estuarios del Tinto y del Odiel.

d) Proyecto Comparado de Geoarqueología Atlántica: en los estuarios del Loira (Francia) y del Guadalquivir (España).

e) Proyecto Comparado de Geoarqueología Atlántica: en la Bahía de Lagos (Algarve, Portugal).

f) Proyecto Comparado de Geoarqueología Atlántica: en el Mar de las Antillas respecto del Valle de Cumanacoa y estuario del río Manzanares (Cumaná, Venezuela).

En el ámbito atlántico-mediterráneo de Andalucía cabe remarcar que los resultados geoarqueológicos buscados pretenden quedar abocados en un conocimiento práctico respecto de las actuales políticas medioambientales que se proyectan en relación con el paisaje físico y humano que hemos heredado (Arteaga y Hoffmann 1999) y en cuya dimensión socio-histórica se encuentra una vez más su realidad futura.

\section{ACERCA DEL PUERTO FENICIO DE GADIR}

\section{Antecedentes preparatorios de la campaña geoarqueológica de Cádiz-2001}

En atención a la apertura renovadora del debate planteado sobre la ubicación de los primeros establecimientos fenicios (Pemán 1941; García Bellido 1945) como unos asentamientos costeros (Estrabón, III 5,3) en el ámbito atlántico-mediterráneo de Andalucía (Schubart 1982) el mejor referente que podemos presentar actualmente en relación con la expectativa de trabajo incentivada también por el prof. Dr. Manuel Pellicer Catalán (Pellicer, Menanteau y Rouillard 1977) queda consignado precisamente en el reciente 
esclarecimiento paleotopográfico realizado en el antiguo casco urbano de Cádiz. Y concretamente, en cuanto al seguimiento geoarqueológico del llamado 'Canal Bahía-Caleta' (Ponce Cordones 1976; 1985) que ha dado por resultado el descubrimiento del Puerto de Gadir (Arteaga et alii 2001).

La importancia científica de este nuevo aporte investigativo, respecto del kothon de Gadir (definiendo su carácter como un puerto cerrado en sí mismo) radica en que la resolución alcanzada entra de lleno en el logro de la desiderata interdisciplinaria planteada desde comienzos y mediados del siglo XX (Gavala 1927; 1959; Pemán 1941; García Bellido 1945) y que sin el concurso de la Geoarqueología venía siendo imposible llegar a mejorar (AA.VV., en Aubet 1994: fig. 63).

Teniendo en cuenta los pormenores metodológicos que publicamos en distintos trabajos de próxima aparición (Arteaga et alii 2001; Arteaga y Roos, en prep.) de una manera resumida podemos reseñar cuáles fueron los antecedentes arqueológicos inmediatos, que llevaron a la realización de la campaña geoarqueológica de Cádiz-2001, y cuáles fueron los resultados que en consecuencia condujeron a la obtención del éxito científico apuntado.

Como hemos reiterado, a tenor de la cartografía existente y de la documentación tradicional antes aludida, era bastante poco cuánto podíamos saber acerca de la posible 'evolución paleo-topográfica' que remanece en la ubicación del actual Puerto de Cádiz. Sobre todo por no contar como ahora (Arteaga et alii 2001) con la referencia de unas adecuadas perforaciones geoarqueológicas, que permitieran establecer con precisión el proceso de colmatación acaecido en el llamado 'Canal Bahía-Caleta' (Ponce Cordones 1976; 1985; Corzo Sánchez 1980; Ramírez Delgado 1982). Y más que nada, que facilitaran contrastar el conocimiento científico de la sedimentación marina que los geólogos y geógrafos sabían que habría comenzado a verificarse en la Bahía de Cádiz, desde el mismo momento en que se estaba formando su 'archipiélago', dados los efectos eustáticos, las mareas y las corrientes oceánicas, que en relación con el cambio del Holoceno se corresponden con el proceso transformador de la fisonomía litoral; referida igualmente en la retracción del nuevo estuario del río Guadalete, en la conformación resultante del 'Caño de Sancti Petri', y en definitiva también en la consolidación de las líneas costeras, continentales e insulares, relativas a la transgresión flandriense (Mörner $1976 ; 1987)$.

Todos estos presupuestos generales, respecto de la Bahía de Cádiz fueron abordados durante el otoño del año 2000 (Schulz et alii, en prep.; Arteaga y Roos, en prep.) sentando las bases investigativas del llamado 'Proyecto Antípolis', gracias a una subvención económica concedida para tales propósitos por parte del Ayuntamiento de San Fernando (Cádiz). Y contando con la colaboración de varios profesores y estudiantes de las Universidades de Bremen, Sevilla y Cádiz, aquellos trabajos preliminares arrojaron unos resultados que en principio facilitaron la preparación y presentación de cinco tesinas de licenciatura en materia de geología, en la Universidad de Bremen (Alemania) por parte de V. Becker, M. Helms, T. Lager, A. Reitz, e I. Wilke, en el año 2001. Y por otro lado, la lectura de una tesina de licenciatura en materia de geoarqueología en la Universidad de Sevilla, por parte de D. Barragán Mallofret, en el mismo año. Estos trabajos parciales habrán de tenerse en cuenta respecto de la amplia bibliografía que comienza a propiciar el 'Proyecto Antípolis' apenas iniciado; y que por otra parte tampoco puede considerarse agotado en el entorno de San Fernando, siendo programadas otras actuaciones entre los años 2003 y 2005 ; incluyendo necesarias perforaciones en el centro mismo de la Bahía, en el reborde costero de Puerto Real, en torno al río Guadalete, y en la zona del Puerto de Santa María.

Durante la campaña inicial del 'Proyecto Antípolis', contando con la colaboración de los entonces tesinandos V. Becker e I. Wilke, y como ellos lo refieren en sus respectivos escritos (Becker 2001; Wilke 2001) los autores firmantes de las consecuentes Memorias Oficiales (Schulz et alii, en prep.; Arteaga y Roos, en prep.) realizaron unos sondeos de prueba en el casco urbano de la ciudad de Cádiz. Se trataba de experimentar la técnica de perforación más adecuada, para ser aplicada durante la próxima campaña que habría de llevarse a cabo en la primavera del año 2001. Se practicaron dos sondeos de prueba (FER 239 
y FER 240). El primero, en la Plaza de la Candelaria (FER 239) buscando observar la colmatación existente en un lugar central del 'Canal Bahía-Caleta'. Y el segundo, en el solar ahora descampado del 'Cine Cómico' de Cádiz, cerca de la Torre de Tavira (FER 240) buscando constatar la potencia estratigráfica depositada en este promontorio, por encima del citado 'Canal' (Ponce 1976; 1985; Corzo 1980; Ramírez Delgado 1982).

En ambas perforaciones se obtuvieron reveladoras experiencias. En la Plaza de la Candelaria, porque se pusieron en evidencia las necesidades técnicas que tendríamos que solventar, para poder cortar en vertical los rellenos constructivos modernos, antes de penetrar en los sedimentos marinos infrapuestos. En el solar del Cine Cómico, porque pudimos comprobar que existía una enorme potencia estratigráfica, incluyendo gruesas capas arenosas, siempre mostrativas de unas actividades antrópicas, algunas de la época romana y otras cuando no más antiguas, augurando llegar hasta niveles fenicios. No habiendo dado con el firme de la roca ostionera, por debajo de las arenas antropizadas, el sondeo hubo de quedar interrumpido por topar la perforadora con una piedra de dimensión considerable, y que resultando alóctona bien podía pertenecer a una insospechada edificación antigua, en aquella interesante orilla del 'Canal', situada en el mismo promontorio de la Torre de Tavira.

Estas expectativas geoarqueológicas del antiguo Cine Cómico abrieron la esperanza de poder encontrar bajo las arenas cobertoras detectadas unos suelos formados por los 'fundadores' fenicios de Gadir.

\section{El cuestionamiento histórico de Gadir y de su localización}

Entrando en la exposición de la campaña geoarqueológica de Cádiz-2001, por todo lo antes apuntado respecto de la Torre de Tavira y del sondeo de prueba practicado (FER 240) en el cruce de la calle San Miguel con la calle Javier de Burgos (Becker 2001; Wilke 2001) hemos de subrayar que desde un principio la objetivación de esta investigación adecuada a la aplicación de una apropiada Geoarqueología Urbana (Arteaga et alii 2001) estaba en gran medida motivada por la desiderata de abordar con ayuda de esta metodología y de sus recursos técnicos el conocimiento y estudio del subsuelo de la ciudad considerada más antigua de Europa Occidental (García Bellido 1985: 361ss.). No era nueva por lo tanto, como también se sabe, la incógnita abierta hasta nuestros días, acerca de la trimilenaria fundación de Gadir por los fenicios. Pero faltaba confirmar a todas luces una relativa concordancia entre las deducciones (1104 a.C.) suministradas a tenor de algunas fuentes escritas (C. Velleius Paterculus, Hist. Rom. 1.2, 3-4) y las que hasta hace bien poco traducían las fuentes arqueológicas (Álvarezy Aranda 1993-94), haciéndose estas últimas reiterativas respecto de los hallazgos pertenecientes a los tiempos púnicos y romanos (Quintero 1917; 1935), como a la inversa resultaban sorprendentes por su parquedad respecto de los hallazgos anteriores propiamente referidos a los fenicios (Pemán 1929; 1959).

En este sentido, no ha dejado de propiciarse una duda metódica, respecto de la ubicación de la Gadir fenicia en el solar de la ciudad de Cádiz, surgiendo propuestas aventurando su posible localización en el asentamiento del Castillo de Doña Blanca, cerca del Puerto de Santa María (Ruiz Mata y Pérez 1995: 126ss.; Ruiz Mata 2001; Pellicer 1996: 148-154).

La campaña geoarqueológica de Cádiz-2001 ha venido a cubrir de una manera bastante exitosa aquella desiderata científica, corroborando con sus resultados estratigráficos en el 'Canal Bahía-Caleta' (Arteaga et alii 2001) la existencia de un Puerto Interior, que perteneciendo a la Gadir fenicia estaba funcionando de una manera continuada cuando menos desde la transición datada hacia el siglo IX-VIII a.C. (Plaza de la Catedral), y durante los tiempos púnicos de la 'Polis Gaditana' (Arteaga 1994; 2001) hasta abarcar la época del Alto Imperio referida a la ciudad de Gades.

En la estratigrafía horizontal obtenida desde el actual Barrio de la Viña, hasta la Plaza de la Candelaria, la colmatación de esta ensenada portuaria no se acelera hasta después del apogeo de la Augustana Urbs 
Iulia Gaditana. Por lo que este resultado coincide plenamente con la cita transmitida por Avieno (O.M., v. 270 ss.), escritor pagano de finales del siglo IV de nuestra Era, cuando exceptuando la solemnidad del templo de Hércules (praeter Herculaneam solennitatem) decía que la "grande y opulenta ciudad en épocas antiguas, ahora pobre, ahora pequeña, ahora abandonada" se había reducido a un campo de ruinas (nunc egena, nunc brevis, nunc destituta, nunc ruinarum ager est).

En efecto, cinco sectores del 'Canal Bahía-Caleta' permitieron establecer la secuencia horizontal de la colmatación, progradante desde el citado Barrio de la Viña, en dirección al Este, hasta formar por delante de la Plaza de la Candelaria un pequeño relicto costero del antiguo brazo de mar, cuyo frente iba a seguir cerrando con su avance en torno a la calle Nueva; mientras que por la parte de la Plaza de San Juan de Dios se estaba elevando la sedimentación formativa de una zona lagunar, predecesora de la llanura de 'La Corredera'. Para finalmente desde el siglo XIV-XV hacia la curvatura que muestra el trazado actual de la Avda. Ramón de Carranza consolidarse una línea de playa, orillando sus fondeaderos portuarios hasta la zona donde se conocen durante el siglo XVI, ya como propios de la Cádiz moderna; es decir, la de la Carrera de Indias, y la del esplendor del setecientos.

\section{El cuestionamiento del llamado paleocauce del 'canal'}

Antes de pasar a comentar los cinco sectores que acabamos de referir a los fondeaderos localizados hasta el momento en el interior del llamado 'Canal Bahía-Caleta' (Arteaga et alii 2001), hemos de remarcar una consideración pertinente a la definición de este 'brazo de mar' más bien como un 'estrecho' formado entre dos islas por efectos de la inundación de estos paleosuelos del quebrado paisaje plioceno-pleistoceno, a partir de la transgresión flandriense.

Se había pensado que el 'Canal Bahía-Caleta' se habría formado debido a la inundación de un paleocauce del río Guadalete (Ponce 1976; 1985; Corzo 1980; Ramírez Delgado 1982; Gracia et alii 2000), observando la coincidencia visual que muestra la actual desembocadura, con la orientación del citado 'Canal' respecto del océano Atlántico (Ramírez Delgado 1982).

No obstante, las perforaciones realizadas por nosotros por detrás de Valdelagrana (Becker 2001; Wilke 2001) muestran que esta proposición resulta imposible. Ya que el encajamiento del paleocauce en esta zona no aparece por lo visto situado a menos de 20 metros, mientras que el firme rocoso plioceno-pleistoceno del 'Canal Bahía-Caleta' no llega jamás a una profundidad mayor por debajo del nivel del mar; cuando un encajamiento como el supuesto tendría que aparecer mucho más bajo en esta zona de la Bahía de Cádiz, en comparación con aquella referida al actual curso del río Guadalete.

Sin entrar para nada en la cuestión formativa del nuevo estuario del río Guadalete, ni en otras relativas a la regresión de la línea costera hasta la conformación periférica de toda la Bahía, desde 'Caño de Sancti Petri' hasta el extremo insular que ahora nos ocupa, nos remitimos por ahora a los resultados obtenidos en las perforaciones preliminares del 'Proyecto Antípolis', para acusar los argumentos contrarios a la proposición referida (Schulz et alii, en prep.; Arteaga y Roos, en prep.), concentrándonos a su vez en las observaciones concernientes a las perforaciones y profundidades ubicadas en relación con el 'Canal BahíaCaleta', y dejando la discusión derivada respecto del nuevo estuario del río Guadalete y sobre la formación del Caño de Sancti Petri para una próxima oportunidad.

Como antes veníamos diciendo, retomando lo apuntado en relación con la estratigrafía horizontal comparada que nos ha permitido establecer el seguimiento de las facies mostrativas de la formación del brazo de mar interinsular (6500 B.P.), de su cegamiento en la parte central de entonces (6500-6300 B.P.) y de la colmatación progresiva de la ensenada abierta a la Bahía, hasta alcanzar la línea costera de la Cádiz moderna, queremos comentar de una manera estratigráfica vertical las secuencias relativas a cinco sectores, del 'Canal BahíaCaleta', para matizar el proceso de su relleno. 


\section{El sector de La Viña}

En el primer sector contrastable, alrededor del Barrio de la Viña, destacan las secuencias verticales obtenidas en las perforaciones CAD 602; CAD 603; CAD 604 y CAD 617.

La perforación CAD 602 muestra que el 'Canal Bahía-Caleta' estaba abierto solamente a comienzos de la existencia del archipiélago gaditano: 6500 B.P. y que no se trataba de un 'canal' en sentido estricto, sino de un brazo de mar como hemos apuntado, situado entre dos islas (Arteaga et alii 2001).

En el extremo Este del estrecho marino, así entendido, había un pequeño islote (CAD 612), que lo dividía en sendos ramales hacia el frente de la Bahía. Un ramal menor penetraba en la dirección de la calle Pelota, hasta la Plaza de la Catedral. Mientras que el ramal mayor entraba hasta la zona de 'Puerto Chico' (CAD 616), y continuaba por la Plaza de la Cruz Verde (CAD 605) hacia la zona de La Viña (fig. 1).

Debido a las acumulaciones arenosas que fueron formando unas 'barras' en los espacios interinsulares de todo el archipiélago (Schulz et alii, en prep.; Arteaga y Roos, en prep.) las islas mayores del mismo quedaron entre ellas conectadas. Hacia el Oeste de la perforación CAD 602, un cegamiento arenoso parecido se produjo como también hemos remarcado entre 6500 y 6300 B.P., aproximadamente.

No por unas causas humanas como algunos autores habían pensado, ni por lo mismo en unos tiempos tan recientes como ellos mismos suponían, el cegamiento del espacio interinsular que ahora nos ocupa habría ocurrido en su tramo medio de entonces (fig. 1) motivando que se originasen dos ensenadas mareales: una abierta a las aguas del océano Atlántico, cerrada hacia la playa de La Caleta, y otra abierta a las aguas de La Bahía, cerrada hacia La Viña. La ensenada marina del Oeste, expuesta a los efectos erosivos del Atlántico, comenzaría a mostrar como el llamado 'frente del vendaval' un desgaste del firme pliocenopleistoceno sumamente remarcado. Por el contrario, la ensenada marina del Este, como el frente que daba a La Bahía, mostraría un proceso de transformación diferente. Ya que al resguardo de los vendavales atlánticos sus rebordes intermareales en lugar de sufrir unos menguantes efectos erosivos conocerían unas acumulaciones sedimentarias, formativas de unas facies más bien progradantes: como la señalada respecto de la colmatación lenta pero constante ocurrida en el citado brazo de mar.

En el sector geoarqueológico analizado con relación a las perforaciones cercanas a La Viña, por lo tanto, mucho antes de que este brazo de mar fuera utilizado como un Puerto Interior por los fenicios de Gadir, se acumularon unos sedimentos mareales producidos por unas aguas relativamente tranquilas; por lo que sabemos que hacia el año 3000 B.P. (Arteaga et alii 2001) resultaba imposible que existiera ninguna conexión marina con el océano Atlántico, a no ser por las 'bocas' de la ensenada abiertas a La Bahía: mucho más amplia y más profunda la del Norte del 'islote' (CAD 612), y mucho más estrecha la que daba a la zona de la Plaza de San Juan de Dios, por esta parte contando con una potencia de agua también menor.

Los fenicios conocieron esta situación estratégica de la ensenada interior, cuando la utilizaron como puerto principal de Gadir, dándole desde un principio una función a todas luces resguardada, respecto de aquella que facultaba la propia condición externa de la ensenada de La Caleta. Desde la Isla de Sancti Petri, hasta las Puntas de San Sebastián y del Nao, el ámbito insular del territorio de Gadir no contaba con un kothon mejor donde guarecer su 'fortaleza', y en esta misma posición marítima por supuesto implantaron el karum más importante de sus dominios occidentales (fig. 2).

La continuidad ocupacional de este 'Puerto Interior' se encuentra demostrada en el sector de La Viña por las cerámicas fenicias aparecidas en la perforación CAD 604. Ya que hasta los tiempos tardopúnicos no se comenzaron a colmatar estos sedimentos propiamente marinos, con las inversiones de las intrusiones estratigráficas que a su vez observamos infrapuestas a los derrubios constructivos romanos del Alto Imperio. La secuencia estratigráfica de La Viña, por consiguiente, muestra a continuación la existencia de un descampado datado a partir de los sedimentos asociados con la Terra Sigillata Clara Africana del siglo IV-V d.C. Por lo que durante la Edad Media esta misma situación se mantiene, y hasta entrado el siglo XVII no se observa 
que unas nuevas actividades edilicias hubieran comenzado a expandirse hacia el Oeste de la actual calle de Sagasta (Arteaga et alii 2001).

Como hemos expuesto en otros trabajos (Arteaga et alii 2001) la formación del descampado 'tardorromano' de La Viña hubo de convertir aquella zona en un suelo utilizado para la agricultura, siendo por lo mismo ocupado acaso por algunas construcciones dispersas, al parecer de una relativa calidad, a tenor de la buena factura de la Terra Sigillata Clara encontrada. Después de los tiempos visigodos, como ya se vislumbraba en los tiempos de Avieno (siglo IV d.C.), el abandono creciente del ámbito urbano de Gades hubo de traducirse en la retracción del núcleo poblacional, hasta verse convertido en una pequeña villa de pescadores, concentrada alrededor del actual Barrio del Pópulo; coincidiendo con la reutilización de los edificios monumentales construidos en los tiempos de los Balbos: como pasaría con el teatro. No sería hasta después de los tiempos medievales, cuando la pequeña medina árabe con sus atarazanas (Martínez Montávez 1974) quedando suplantada por la villa cristiana de Alfonso X el Sabio (siglo XIII) hubiera comenzado a resurgir, creciendo después hacia los arrabales de Santa María y de Santiago (siglo XV) alrededor de la Plaza de la Corredera. En el extremo del arrabal de Santiago, hacia los tiempos de Carlos V había una cerca de cierre que daba a La Viña, coincidiendo por lo mismo con el trazado curvo que ahora mantiene el propio de la citada calle de Sagasta.

En definitiva, la mención reiterada de la calle de Sagasta, respecto de La Viña que existía por la parte exterior de la cerca de cierre del Barrio de Santiago, sirve para matizar la manera en que aquellos descampados hasta las construcciones del Barrio del Nuevo Mundo (La Viña) no fueron otra vez urbanizados de una manera decisiva, dándose esta circunstancia por lo tanto a partir de entrado el siglo XVII, como lo muestran las cerámicas y los restos de materiales de edificaciones destruidas por otras posteriores, según las perforaciones CAD 603, CAD 604 y CAD 617. Es decir, por las nuevas edificaciones que siendo pertenecientes al citado esplendor gaditano del setecientos, hasta en sus barrios más humildes dejaban para siempre soterradas las evidencias históricas de otras pasadas grandezas.

\section{El sector de 'Puerto Chico'}

En el segundo sector de las perforaciones, desde la Plaza de la Cruz Verde (CAD 605) hasta el Mercado Central (CAD 615) y la calle Puerto Chico (CAD 616), la profundidad del agua se hace relativamente mayor y la apertura navegable de la ensenada de La Bahía también dura mucho más que hacia La Viña. En el fondeadero localizado en 'Puerto Chico', por ejemplo, los sedimentos marinos ubicados a unos seis metros de profundidad (sin llegar al firme) estuvieron asociados a numerosos fragmentos de la cerámica Campaniense A Tardía: perteneciendo por lo tanto al tiempo de los Balbos.

Este dato homologado también en las perforaciones realizadas desde la Plaza de la Catedral (CAD 613) hasta la zona del Mercado Central (CAD 615) confirma que los entornos portuarios formados por estos fondeaderos contaban con una potencia de agua suficiente para permitir durante el Alto Imperio que penetraran todavía unas naves de buen calado, hasta el mismo corazón de Gades (Arteaga et alii 2001).

Una prueba fehaciente de que la orilla marina del reborde costero llegaba durante el Alto Imperio hasta la zona del Mercado Central, la tenemos en la secuencia estratigráfica obtenida entre las perforaciones CAD 606 y CAD 615 (Arteaga et alii 2001), así como también en las instalaciones de las piscinas de salazones ubicadas en el vecino solar del antiguo Teatro Andalucía (Cobos, Muñoz y Perdigones 1995-96).

La orilla marina de este reborde costero, desde la Plaza de las Flores (CAD 614) daba un giro hacia el Nordeste, para pasando por delante del 'Palillero' y la calle Columela buscar la costa en la dirección de la Aduana, por la Iglesia de San Agustín. No se trataba de un reborde regular, como el de un 'Canal', sino más bien de un reborde accidentado, con unos giros acusados por el firme plioceno-pleistoceno: como ocurría también con el reborde costero que iba desde la Plaza de la Catedral (calle Pelota) hacia 'Puerto Chico'. 
El referente geoarqueológico de 'Puerto Chico', permite matizar algunas afirmaciones que se venían propugnando en relación con la supuesta colmatación de esta zona del 'Canal' en tiempos anteriores a los Balbos (Ramírez Delgado 1982). Ya que una vez confirmada su apertura, hasta mucho más tarde, carece de sentido pensar que por causa de aquel supuesto 'cegamiento' Balbo el Menor habría promovido la construcción de una 'Nueva Gades'.

Entendemos que la política expansionista del urbanismo de Gades, sin perder la noción articulada alrededor de su Puerto Interior, comportaría más bien un crecimiento integral (Arteaga et alii 2001). Por lo que la evergética propagandística de la oligarquía naviera y comercial gaditana, remodelando grandes espacios de la vieja ciudad entonces convertida en Municipium Civium Romanorum (T. Livius, perioch. 110; Dio Cassius, XLI 24) hubo de correr pareja con la urbanística de una ordenación 'gemela' concebida como Didýme (Estrabón, III 5,3) alrededor del espacio dividido por el puerto, para albergar en aquella 'Nueva Gades' una masa de población creciente.

Desde esta misma perspectiva igualmente pareja sería al lado de las ricas villas residenciales que continuamos detectando en el entorno de San Fernando (Barragán 2001) la estructuración rural de los nuevos suelos salineros, de las vecinas almadrabas, y de los embarcaderos que adscribimos a los alfares y hornos excavados por nosotros mismos en Antípolis (Arteaga et alii 1997); sin olvidar por entonces la construcción por Balbo de un astillero en la parte frontera de la tierra firme, ni la infraestructura concerniente al acueducto, ni la mención referencial del gran teatro de Gades (Cicero, Ad fam. X 32,2) que hacia el año 43 a.C. ya estaba destinando sus catorce gradas primeras al asiento de unos quinientos caballeros; siendo este testimonio suficiente para explicar las adquisiciones de tierras que aquellas ricas familias gaditanas expandieron por los alrededores de La Bahía; para como unos propietarios fundiarios acrecentar sus méritos honoríficos, en cuanto que además eran requeridos para poder disfrutar el derecho de ingresar en el ordo senatorial.

En definitiva, podemos concluir a tenor de la secuencia de 'Puerto Chico' que el puerto fenicio de Gadir, había continuado siendo utilizado hasta los tiempos de la Augustana Urbs Iulia Gaditana citada por Plinio el Viejo (Nat. hist. IV 119-120) y hasta mucho más tarde; como podemos establecer en comparación con la retracción urbana acaecida a partir del siglo III d.C. (Avieno, O.M., siglo IV d.C.). Siendo así por lo que tampoco puede cuestionarse su inmejorable situación estratégica durante la época floreciente de la 'Polis Gaditana' (Arteaga 1994).

Cabe añadir otra matización geoarqueológica respecto de la sedimentación marina documentada en el fondeadero de 'Puerto Chico', y ella se refiere a la imposibilidad de que existiera otra salida navegable desde aquí hacia las aguas del océano Atlántico.

De haberse dado esta apertura, como suponen algunos autores, los sedimentos depositados en el fondo de la perforación CAD 616 no hubieran presentado una granulometría tan fina; siendo por lo tanto propia de unas aguas relativamente tranquilas.

El promontorio que se levanta por el Sur de dicha perforación estaba elevado unos 9 metros por encima del nivel del mar, por lo que la navegación hasta dicho fondeadero solamente se podía llevar a cabo viniendo desde La Bahía.

\section{El sector portuario de la Plaza de la Catedral}

El tercero de los sectores que podemos referir a las perforaciones realizadas durante la campaña geoarqueológica de Cádiz-2001 (Arteaga et alii 2001) se encuentra ubicado alrededor del fondeadero localizado en torno a la Plaza de la Catedral (CAD 613).

Constituye este lugar (fig. 1) sin duda alguna la zona portuaria que más y mejores resultados ha aportado hasta el momento: respecto de la Gadir fenicia, y respecto de su continuidad durante los tiempos púnicos 
de la 'Polis Gaditana' (Arteaga 1994) hasta conectar finalmente con el crecimiento urbanístico del Municipium Civium Romanorum de Gades: la Didýme de Estrabón (III 5,3).

En la perforación CAD 613 (Arteaga et alii 2001) realizada en la mañana del 30 de Marzo del año 2001, en unas profundidades comprendidas entre los 7'00 y los 7' 50 metros, y referidas todavía a unos 2' 00 metros de potencia de agua marina, aparecieron unos sedimentos antropizados, con grava fina, con arena, con mucho limo, y con piedras, asociados también con diversos fragmentos de cerámica fenicia (fig. 3a-e).

Destacaría entre todos ellos un fragmento amorfo de pasta compacta, de buena calidad, de color marrón rojizo, con la superficie exterior cubierta por completo de barniz rojo. La pared globular del fragmento denota que se trata de un 'oenochoe' de panza abombada, de tipología arcaica propia del siglo IX-VIII a.C. (fig. 3a). Otros pequeños cuencos, de paredes finas, también fenicios (fig. 3b-c) pudieron quedar estratificados en estos mismos sedimentos, al igual que algunos fragmentos anfóricos de pasta beige anaranjada, con inclusiones de color granate, y con la cara exterior cubierta por un baño arcilloso de color amarillento (fig. 3e) recordando producciones similares encontradas en Cartago. Otros fragmentos con unas pastas no esquistosas, como las conocidas en Andalucía, pero igualmente de buena calidad y muy compactas, como vemos en el 'oenochoe' de barniz rojo, bien pueden pertenecer a unas cerámicas procedentes de Oriente, cuando no de la propia Fenicia. Pensamos que el contexto en que aparecen ubicados estos fragmentos cerámicos, al lado de otros hechos también a torno, aunque de más difícil clasificación (amorfos) resulta concluyente respecto de la utilización portuaria de este fondeadero interior, por parte de los barcos fenicios, alrededor del siglo IX-VIII a.C. cuando menos. No se trata de unas fechas absolutas, por lo que algún día calibradas pueden aportar unas interesantes matizaciones, cuando contemos con otras muestras apropiadas para las dataciones del Carbono-14 que ahora sabemos dónde buscar.

No hemos llegado a extraer unas muestras arqueológicas más profundas, aunque hasta los 7' 50 y 8'00 metros (casi 3'00 por debajo del nivel del mar) continuaron apareciendo esquirlas de hueso y partículas minúsculas de cerámica. La expectativa cronológica inicial, por lo tanto, queda abierta; pero ya no puede ponerse en duda que hemos dado con los sedimentos marinos más antiguos del Puerto Fenicio de Gadir (Arteaga et alii 2001).

Nuestro resultado geoarqueológico de Cádiz-2001 viene a cubrir con creces la expectativa alimentada con el sondeo de prueba (FER 240) practicado en el otoño del año 2000, en el solar del Cine Cómico. Por lo que las noticias fortuitas sobre otros hallazgos sueltos, relativos a cronologías de los siglos VIII-VII a.C. (Pemán 1929; 1959) comienzan a completarse a los lados Norte (Torre de Tavira), Sur (Casa del Obispo), Este (el puerto) y Oeste (La Viña) del propio 'Canal Bahía-Caleta', siendo todo ello comprensible desde la más alta datación que cabe esperar para tales ocupaciones insulares, mediadas por el principal espacio portuario situado entre dicha zona del 'Palillero', la zona de 'Puerto Chico', y la zona de la 'Catedral', aquí con dataciones que cuando menos remontamos al siglo IX-VIII a.C. (Arteaga et alii 2001).

Entendemos de esta manera que no existiendo previamente ningún centro de poder tartesio directamente asentado en el ámbito insular (Arteaga 2001) los fenicios ocupando este espacio marítimo y portuario se establecieron teniendo por frente a los pobladores de la tierra firme, en aquella 'periferia' territorial de Tarsis. Por lo que sin dudar que en los centros tartesios se integraron muchos elementos venidos de Oriente con los fenicios, y que en los sitios fenicios habitaron muchos 'indígenas', pensamos que la identificación tartesia de unos núcleos poblacionales tan importantes como eran los existentes en el Cabezo de San Pedro (Huelva) y en el Castillo de Doña Blanca, además de aquellos que se hallaban localizados en el Valle del Guadalquivir (Arteaga y Roos 1992; 1995) no debe para nada ponerse en duda; ni debe confundirse con la identificación oriental de los fundadores de Gadir.

Antes de la fundación portuaria de Gadir, para nosotros resulta evidente que correspondería a los tartesios el precedente económico-político de haber estado explotando también la estructuración territorial 'isleña', sin que este 'presupuesto' hubiera obstaculizado el hecho de que los fenicios, una vez conocedores de las 
costas mediterráneas de Sexi, y una vez conocedores de las costas atlánticas de Onoba, como todavía aludía Estrabón (III 5,5) se hubieran acabado asentando en el territorio insular de Gadir, a todas luces más favorable. Para desde allí consolidar una política económica en común con los principales centros tartesios del mediodía peninsular, situados sobre todo en la Baja Andalucía.

En esta expectativa fenicia-tartesia, pensamos nosotros que contando con la connivencia de los segundos los primeros citados acabaron afincando su karum capital en una especie de kothon natural sumamente estratégico (fig. 2). Es decir, en un puerto cerrado en sí mismo; como sería el vinculado al lugar llamado Gadir, para de esta forma mantener una doble política estatal: por un lado respecto de Tiro, y por otro lado respecto de Tarsis (Arteaga et alii 2001).

\section{El sector portuario de La Corredera}

El cuarto sector que podemos matizar espacialmente de acuerdo con las perforaciones geoarqueológicas realizadas (Arteaga et alii 2001) se encuentra ubicado alrededor de la actual Plaza de San Juan de Dios, conocida antes como Plaza de Isabel II, y desde la transición al siglo XVI como Plaza de la Corredera.

Tiene este espacio colindante con La Bahía un interés muy especial. Primero, porque se hallaba presidido desde la transgresión flandriense por una pequeña isla (CAD 612), que como hemos dicho separaba en sendos ramales al brazo de mar que se hallaba entre las dos islas mayores: la del promontorio de la Torre de Tavira hacia el Norte, y la del promontorio de la Catedral hacia el Sur.

En segundo lugar, porque a partir del cegamiento de este brazo de mar (6500-6300 B.P.) por la zona originaria de la playa de La Caleta (fig. 1) este pequeño islote pasaría a constituir un punto referente de navegación, por un lado, respecto del ramal septentrional (más ancho y más profundo) que penetraba desde los fondeaderos orillados a La Bahía en dirección a 'Puerto Chico' (fig. 1); y por otro lado, respecto del ramal meridional que penetrando precisamente en la dirección de la calle Pelota resultaba confluyente en la zona de la Plaza de la Catedral (CAD 613).

En tercer lugar, el interés que tiene el espacio situado por delante del pequeño islote en los tiempos de Gadir (3000 B.P.) radica en que teniendo una relativamente menor profundidad de agua (Arteaga $e t$ alii 2001) en comparación con el fondeadero interior situado en la Plaza de la Catedral (fig. 1) conformaba una especie de antesala marítima entre La Bahía y la citada zona portuaria (CAD 613).

Durante los tiempos de Gadir, no sabemos a ciencia cierta si cualquier forma de navegación era posible por el ramal marino que pasando por el Sur de la pequeña isla llegaba a conectar con la zona del fondeadero situado en la Plaza de la Catedral: aunque damos por supuesto que las naves que penetraban por la parte del ramal septentrional pudieron en cualquier caso tener siempre un mayor calado que aquellos barcos que orillaban por delante de la calle Pelota, desde la zona de San Juan de Dios.

Dada la menor profundidad del firme detectada mediante las perforaciones practicadas en este espacio frontero que miraba a La Bahía (CAD 608; CAD 609), y dadas las curvas de nivel (CAD 607) que incluso de una manera submarina giraban para conectar con el islote que afloraba sobre el nivel del agua, en función de este 'istmo' sumergido, formado por el firme plioceno-pleistoceno, por delante de la 'Puerta del Pópulo' se remarcaba la curvatura de un reborde costero; contra el cual los efectos mareales propiciaron un proceso de colmatación, progradante hacia La Bahía, y que al elevarse iría conformando la aparición de una llanada costera, antecesora de aquella que cada vez más desecada daría lugar al suelo donde se asentaría la Plaza de la Corredera (Horozco 1591; 1598).

El proceso de colmatación causado por este firme plioceno-pleistoceno, alrededor de la isla-península detectada entre la perforación CAD612 y la curva de nivel referidad al Barrio del Pópulo, esperamos precisarlo 
mejor cortando por el 'istmo' un perfil estratigráfico, mediante unos nuevos sondeos alineados en la dirección de la calle Pelota. Por lo que esta desiderata, mientras se mantenga abierta, recomienda que expresemos una duda metódica acerca de la 'navegación' por el brazo marino situado entre la Plaza de San Juan de Dios y la Plaza de la Catedral; sin que en ningún caso el resultado geoarqueológico definitivo reste importancia a la matización del calado de los barcos que podían orillarse hacia el reducto costero de la 'Puerta del Pópulo', en comparación con el gran calado que tenían aquellas naves que penetraban por el ramal marino septentrional, llegando hasta los inmejorables fondeaderos del Puerto Interior de Gadir.

Nosotros damos por descontado, por consiguiente, que en la elevación aflorante del 'pequeño islote' los navegantes que llegaban al Puerto Interior desde las aguas de La Bahía contaban con un excelente punto de referencia, por lo que nada extrañaría que esta condición estuviera de alguna manera señalizada, como en otros tiempos hicieron los 'faros' gaditanos.

\section{El sector central del puerto natural de Cádiz}

El quinto sector que matizamos respecto de las perforaciones geoarqueológicas realizadas (Arteaga et alii 2001) podemos referirlo al entorno de la Plaza de la Candelaria (CAD 601); y a la zona situada entre la Iglesia de San Agustín (CAD 618) y la Plaza Mendizabal (CAD 619): para así entender la secuencia estratigráfica documentada hacia el Oeste de la calle Nueva, y hacia el Este de esta misma calle (fig. 1).

El trazado recto que muestra la calle Nueva, respecto del trazado curvo que muestra la Avda. Ramón de Carranza (CAD 611), permite ilustrar la referencia de un antes y un después respecto del aprovechamiento del nuevo suelo formado por el proceso de colmatación, que hacia la Edad Media avanzaba por la Plaza de la Candelaria (CAD 601), rellenando luego hasta La Bahía el último relicto que quedaba del antiguo brazo de mar.

En este proceso de colmatación alineado entre la perforación CAD 601 y la CAD 611 de la Avda. Ramón de Carranza (fig. 1) contando con la delimitación de los rebordes detectados cerca de la Iglesia de San Agustín (CAD 618) y de la Plaza Mendizabal (CAD 619) pudimos documentar con precisión la progradación formativa de una playa costera que conectando con la propia de la 'Plaza de la Corredera' acabaría orillando con ella misma la ubicación del 'Puerto de Cádiz' hacia los fondeaderos del frente de La Bahía, consolidando en estos últimos la situación que iba a conocer durante los tiempos modernos (fig. 1).

En conclusión, podemos observar que desde los alrededores del actual 'Barrio de la Viña' los fenicios conocieron una ensenada marina abierta por dos ramales hacia la Bahía (fig. 2), y que contando entonces con la indicación del pequeño islote situado entre ambos pudieron hacer llegar unas naves de mayor calado hasta los fondeaderos portuarios localizados entre la Plaza de la Catedral, 'Puerto Chico' y la Torre de Tavira (fig. 2). Teniendo en cuenta la estratigrafía comparada obtenida mediante las perforaciones practicadas hacia el Oeste de la Plaza de la Candelaria, este Puerto Interior continuaba durante el Alto Imperio siendo utilizado; dando sentido al crecimiento 'gemelo' de la Didýme referida por Estrabón (III 5,3).

En suma, permitiendo todo lo antes apuntado afirmar que en esta especie de kothon el famoso karum de Gadir, a través de los tiempos de la 'Polis Púnica Gaditana', consigna una continuidad histórica que conecta de una manera irrefutable con el Puerto Magno de la Augustana Urbs Iulia Gaditana citada por Plinio el Viejo, y cuya pasada grandeza recordaría con nostalgia el poeta Rufo Festo Avieno. 


\section{BIBLIOGRAFÍA}

ÁLVAREZ ROJAS, A. y ARANDA LINARES, C. (1993-94): Bibliografía de Cádiz en época fenicia y romana", Boletín del Museo de Cádiz 6: 53-66. Cádiz.

ARRIBAS, A. y ARTEAGA, O. (1975): El yacimiento fenicio de la desembocadura del río Guadalhorce (Málaga), Cuadernos de Prehistoria de la Univ. de Granada, Serie Monográfica 2. Granada.

ARTEAGA, O. (1988): "Zur phönizischen Hafensituation von Toscanos. Vorbericht über die Ausgrabungen in Schnitt 44". En: Forschungen zur Archäologie und Geologie im Raum von Torre del Mar 1983/84. Madrider Beiträge 14: 127-141. Mainz.

— (1994): "La Liga Púnica Gaditana". En: VIII Jornadas de Arqueología Fenicia-Púnica. Trabajos del Museo Arqueológico de Ibiza 33: 23-57. Ibiza.

_ (2001): "La emergencia de la 'Polis' en el mundo púnico occidental". En: M. ALMAGRO et alii: Protohistoria de la Península Ibérica: 217-281. Barcelona.

ARTEAGA, O. y HOFFMANN, G. (1999): "Dialéctica del proceso natural y sociohistórico en las costas mediterráneas de Andalucía", Revista Atlántica-Mediterránea de Prehistoria y Arqueología Social 2: 13-121. Cádiz.

ARTEAGA, O. y ROOS, A.M. (1992): "El Proyecto Geoarqueológico de las Marismas del Guadalquivir. Perspectivas arqueológicas de la campaña de 1992”, Anuario Arqueológico de Andalucía 1992-II: 329-339. Sevilla.

(1995): "Geoarchäologische Forschungen im Umkreis der Marismas am Río Guadalquivir (Niederandalusien)", Madrider Mitteilungen 36: 199-218. Mainz.

_ (en prep.): "Geoarchäologische Forschungen in der Bucht von Cádiz", Madrider Mitteilungen (en preparación). Mainz.

ARTEAGA, O. y SCHULZ, H.D. (2000): "El puerto fenicio de Toscanos. Investigación geoarqueológica en la costa de la Axarquía (Vélez-Málaga 1983/84). Instituto Arqueológico Alemán", En: T. ÑACO, O. ORESTI y A. PRIETO (eds.), European Comission. COST Action G2. Anàlisis paleoambientals i estudi del territori: 13-47. Barcelona.

ARTEAGA, O.; SCHULZ, H.D. y ROOS, A.M. (1995): “El problema del 'Lacus Ligustinus'. Investigaciones geoarqueológicas en torno a las Marismas del Bajo Guadalquivir”. En: Tartessos 25 años después 1968 1993. Actas del Congreso Conmemorativo del V Symposium Int. de Prehistoria Peninsular: 99-135. Jerez de la Frontera.

ARTEAGA, O.; HOFFMANN, G.; SCHUBART, H. y SCHULZ, H.D. (1985): “Investigaciones geológicas y arqueológicas sobre los cambios de la línea costera en el litoral de la Andalucía mediterránea. Informe preliminar (1985)", Anuario Arqueológico de Andalucía 1985-II: 117-122. Sevilla.

ARTEAGA, O. et alii (1988): Forschungen zur Archäologie und Geologie im Raum von Torre del Mar 1983/84. Madrider.Beiträge 14. Mainz.

ARTEAGA, O.; CASTAÑEDA, V.; HERRERO, N. y PÉREZ RODRÍGUEZ, M. (1997): "Los hornos tardopúnicos de Torre Alta (San Fernando, Cádiz). Excavación de Urgencia de 1997", Anuario Arqueológico de Andalucía 1997-III: 128-136. Sevilla.

ARTEAGA, O.; KÖLLING, A.; KÖLLING, M.; ROOS, A.M.; SCHULZ, H. y SCHULZ, H.D. (2001): "Geoarqueología Urbana de Cádiz". Anuario Arqueológico de Andalucía 2001 (en prensa). Sevilla.

AUBET, M.E. (1994): Tiro y las colonias fenicias de Occidente. $2^{a}$ edición. Barcelona.

BARRAGÁN MALLOFRET, D. (2001): Investigación geoarqueológica en San Fernando. Cádiz. Memoria de Licenciatura inédita. Departamento de Prehistoria y Arqueología de la Universidad de Sevilla.

BECKER, V. (2001): Entwicklung der Küstenlinie im Holozän in der Bucht von Cádiz. Memoria de Licenciatura inédita. Fachbereich Geowissenschaften der Universiät Bremen. 
COBOS RODRÍGUEZ, L.; MUÑOZ VICENTE, A. y PERDIGONES MORENO, L.(1995-96): “Intervención arqueológica en el solar del antiguo Teatro Andalucía de Cádiz: la factoría de salazones y la representación gráfica del faro de Gades", Boletín del Museo de Cádiz 7: 115-132. Cádiz.

CORZO SÁNCHEZ, R. (1980): "Paleotopografía de la bahía gaditana", Gades 5: 5-14. Cádiz.

GARCÍA Y BELLIDO, A. (1945): España y los españoles hace dos mil años según la 'Geografia' de Strábon. Madrid.

- (1985): Urbanística de las grandes ciudades del Mundo Antiguo. $2^{\text {a }}$ edición. Madrid.

GAVALA Y LABORDE, J. (1927): "Cádiz y su bahía en el transcurso de los tiempos geológicos", Boletín del Instituto Geológico y Minero de España 49 ( 9 de la $3^{\text {a }}$ serie). Madrid.

- (1959): La Geología de la Costa y Bahía de Cádiz y el poema 'Ora Maritima' de Avieno. Madrid.

GRACIA, F.J.; ALONSO, C.; GALLARDO, M.; GILES, F.; BENAVENTE, J. y LÓPEZ-AGUAYO, F. (2000): "Evolución eustática postflandriense en las marismas del Sur de la Bahía de Cádiz", Geogaceta 27: 71-74.

GRAS, M.; ROUILLARD, P. y TEIXIDOR, J. (1989): L'univers phénicien. Paris.

HOFFMANN, G. (1988): Holozänstratigraphie und Küstenlinienverlagerung an der andalusischen Mittelmeerküste. Berichte aus dem Fachbereich Geowissenschaften der Universität Bremen 2. Bremen.

HOROZCO, A. de (1591): "Discurso de la fundación y antiguiedades de Cádiz y los demás subcesos que por ella han pasado". Reimpresión en Documentos inéditos para la historia de Cádiz. 1929. Cádiz.

- (1598): Historia de la ciudad de Cádiz. Reimpresión 1845. Cádiz.

MARTÍNEZ MONTÁVEZ, P. (1974): Perfil del Cádiz hispanoárabe. Cádiz.

MENANTEAU, L. (1982): Les Marismes du Guadalquivir. Exemple de transformation d'un paysage alluvial au cours du Quaternaire récent. Tesis Doctoral. Université de Paris-Sorbonne. Paris.

MÖRNER, N.A. (1976): "Eustasy and geoid changes", Journal of Geology 84: 123-151. Chicago.

(1987): "Models of global sea level changes", en M.J. TOOLEY e I. SHENNAN (eds.): Sea Level Changes: 332-355. Oxford.

PELLICER CATALÁN, M. (1962): Excavaciones en la necrópolis púnica 'Laurita' del Cerro de San Cristóbal (Almuñécar, Granada), Excavaciones Arqueológicas en España 17. Madrid.

___(1996): "Estrategia de los asentamientos fenicios en Iberia", Boletín de la Real Academia de Bellas Artes de Santa Isabel de Hungría 24: 143-167. Sevilla.

PELLICER, M.; MENANTEAU, L. y ROUILLARD, P. (1977): "Para una metodología de localización de colonias fenicias en las costas ibéricas: el Cerro del Prado", Habis 8: 217-251. Sevilla.

PEMÁN, C. (1929): "Figurilla de bronce hallada en Cádiz", Boletín del Museo de Bellas Artes de Cádiz 13: 7-15. Cádiz.

- (1941): El pasaje tartéssico de Avieno a la luz de las últimas investigaciones. Madrid. (1959): "El capitel de tipo protojónico de Cádiz", Archivo Español de Arqueología 32: 58-70. Madrid.

PONCE CORDONES, F. (1976): “Consideraciones en torno a la ubicación del Cádiz fenicio", Suplemento Diario de Cádiz. 12 de Diciembre de 1976. Cádiz.

- (1985): "Consideraciones en torno a la ubicación del Cádiz fenicio", Anales de la Universidad de Cádiz 2: 99-121. Cádiz.

QUINTERO, P. (1917): Cádiz, primeros pobladores: hallazgos arqueológicos. Cádiz.

- (1935): Excavaciones en Cádiz. Memoria de la Junta Superior de Excavaciones y Antigüedades 134. Madrid.

RAMÍREZ DELGADO, J.R. (1982): Los primitivos núcleos de asentamiento en la ciudad de Cádiz. Cádiz. RUIZ MATA, D. (2001): "Tartessos", en M. ALMAGRO et alii: Protohistoria de la Península Ibérica: 1-185. Barcelona. 
RUIZ MATA, D. y PÉREZ, C.J. (1995): El poblado fenicio del Castillo de Doña Blanca (El Puerto de Santa María, Cádiz). El Puerto de Santa María.

SCHUBART, H. (1982): “Asentamientos fenicios en la costa meridional de la Península Ibérica”, Huelva Arqueológica 6: 71-99. Huelva.

SCHUBART, H.; NIEMEYER, H.G. y PELLICER CATALÁN, M. (1969): Toscanos. La factoría paleopúnica en la desembocadura del río de Vélez. Excavaciones de 1964. Excavaciones Arqueológicas en España 66. Madrid.

SCHUBART, H.; ARTEAGA, O.; HOFFMANN, G. y KUNST, M. (1988): "Investigación geológicaarqueológica sobre la antigua línea de costa en Andalucía. Campaña 1988", Anuario Arqueológico de Andalucía, 1988-II: 185-189. Sevilla.

SCHÜLE, W. (1986): "El Cerro de la Virgen de la Cabeza, Orce (Granada): Consideraciones sobre su marco ecológico y cultural", en Homenaje a Luis Siret 1934-1984: 208-220. Sevilla.

SCHULZ, H.D. (1983): "Zur Lage holozäner Küsten in den Mündungsgebieten des Río de Vélez und des Río Algarrobo (Málaga). Vorbericht”, Madrider Mitteilungen 24: 59-64. Mainz.

- (1988): "Geologische Bearbeitung der Grabung in der 'Hafenbucht' von Toscanos", en Forschungen zur Archäologie und Geologie im Raum von Torre del Mar 1983/84. Madrider Beiträge 14: 142-154. Mainz.

SCHULZ, H.D.; JORDT, K.P. y WEBER, W. (1988): "Stratigraphie und Küstenlinien im Holozän (Río de Vélez)", en Forschungen zur Archäologie und Geologie im Raum von Torre del Mar 1983/84. Madrider Beiträge 14: 5-38. Mainz.

SCHULZ, H.D.; FELIS, T.; HAGEDORN, C.; LÜHRTE, R. VON; REINERS, C.; SANDER, H.; SCHNEIDER, R.; SCHUBERT, J. y SCHULZ, H. (1992): "La línea costera holocena en el curso bajo del río Guadalquivir entre Sevilla y su desembocadura en el Atlántico. Informe preliminar sobre los trabajos de campo realizados en Octubre y Noviembre de 1992", Anuario Arqueológico de Andalucía 1992-II: 323-327. Sevilla.

SCHULZ, H.D.; FELIS, T.; HAGEDORN, C.; LÜHRTE, R. VON; REINERS, C.; SANDER, H.; SCHNEIDER, R.; SCHUBERT, J. y SCHULZ, H. (1995): "Holozäne Küstenlinie am Unterlauf des Río Guadalquivir zwischen Sevilla und der Mündung in den Atlantik", Madrider Mitteilungen 36: 219-232. Mainz.

SCHULZ, H.D.; BARRAGÁN MALLOFRET, D.; BECKER, V.; LAGER, T.; HELMS, M.; REITZ, A. y WILKE, I. (en prep.): "Geschichte des Küstenverlaufs in der Bucht von Cádiz und San Fernando im Holozän", Madrider Mitteilungen (en preparación). Mainz.

WILKE, I. (2001): Die Bucht von Cádiz im Holozän. Memoria de Licenciatura inédita. Fachbereich Geowissenschaften der Universität Bremen. 


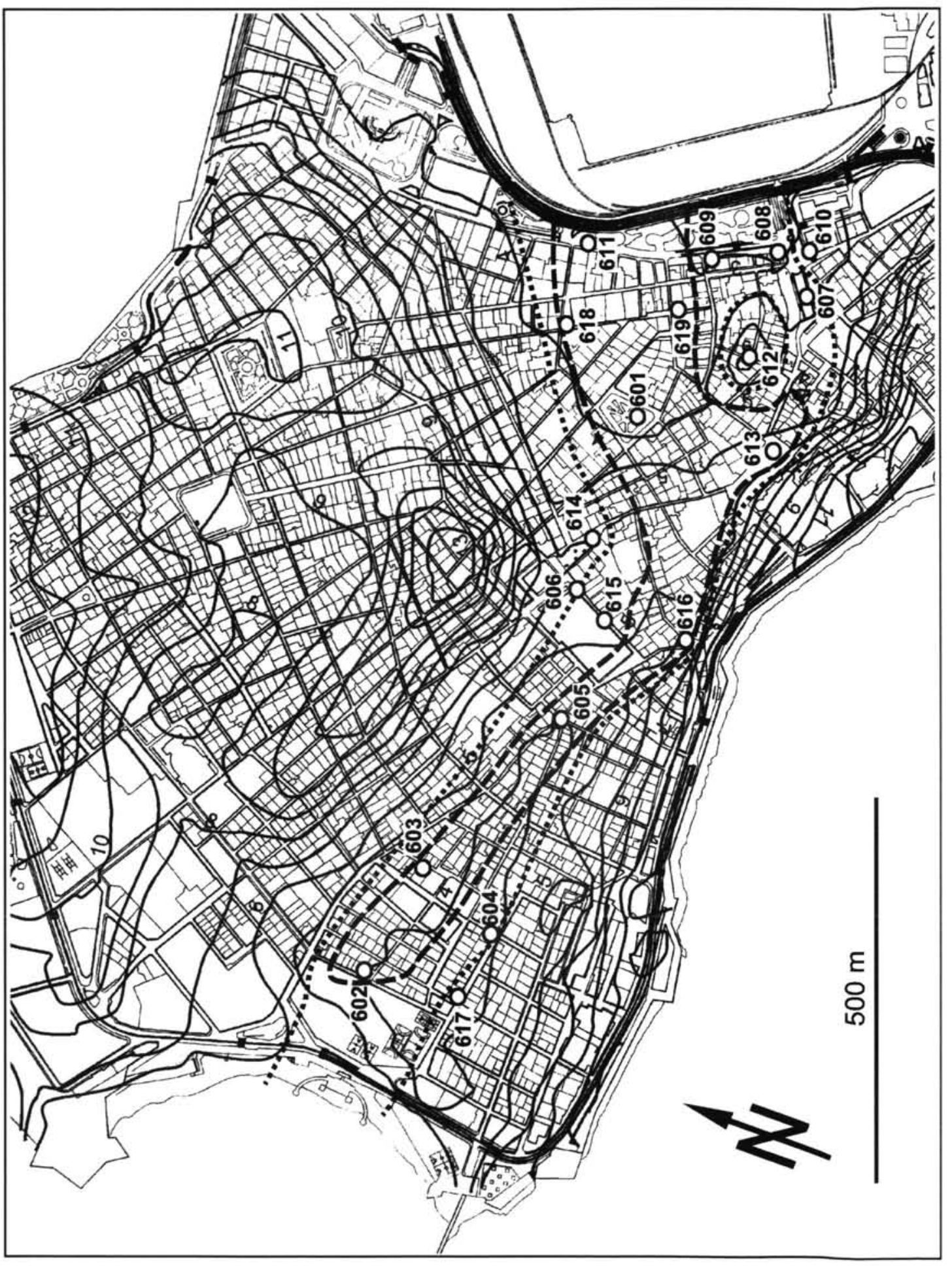

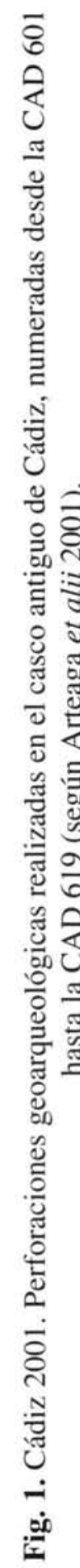




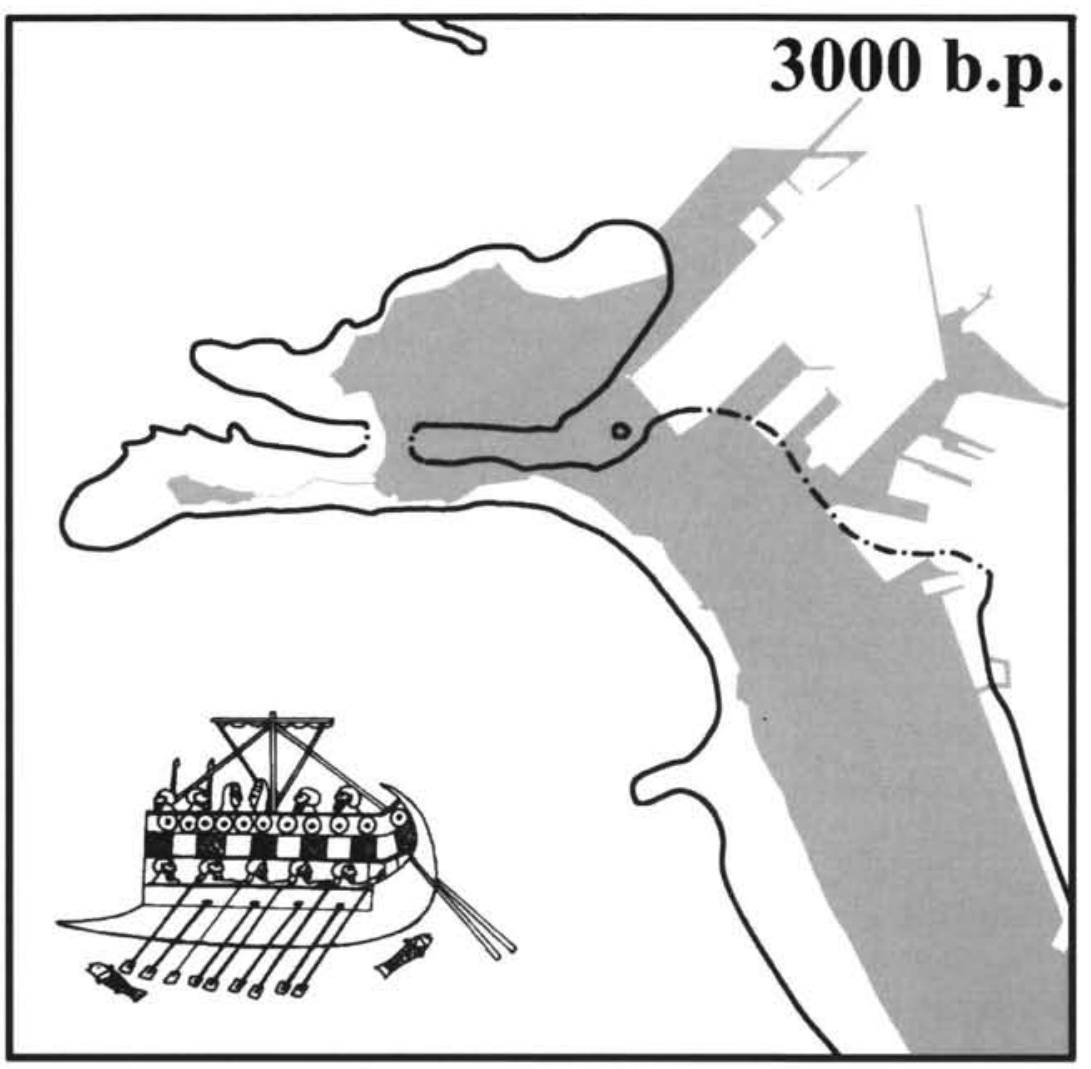

Fig. 2. Cádiz 2001. Reconstrucción de la línea de costa alrededor del año 3000 B.P. En trama gris el estado actual de la 'península' gaditana (según Arteaga et alii 2001). 

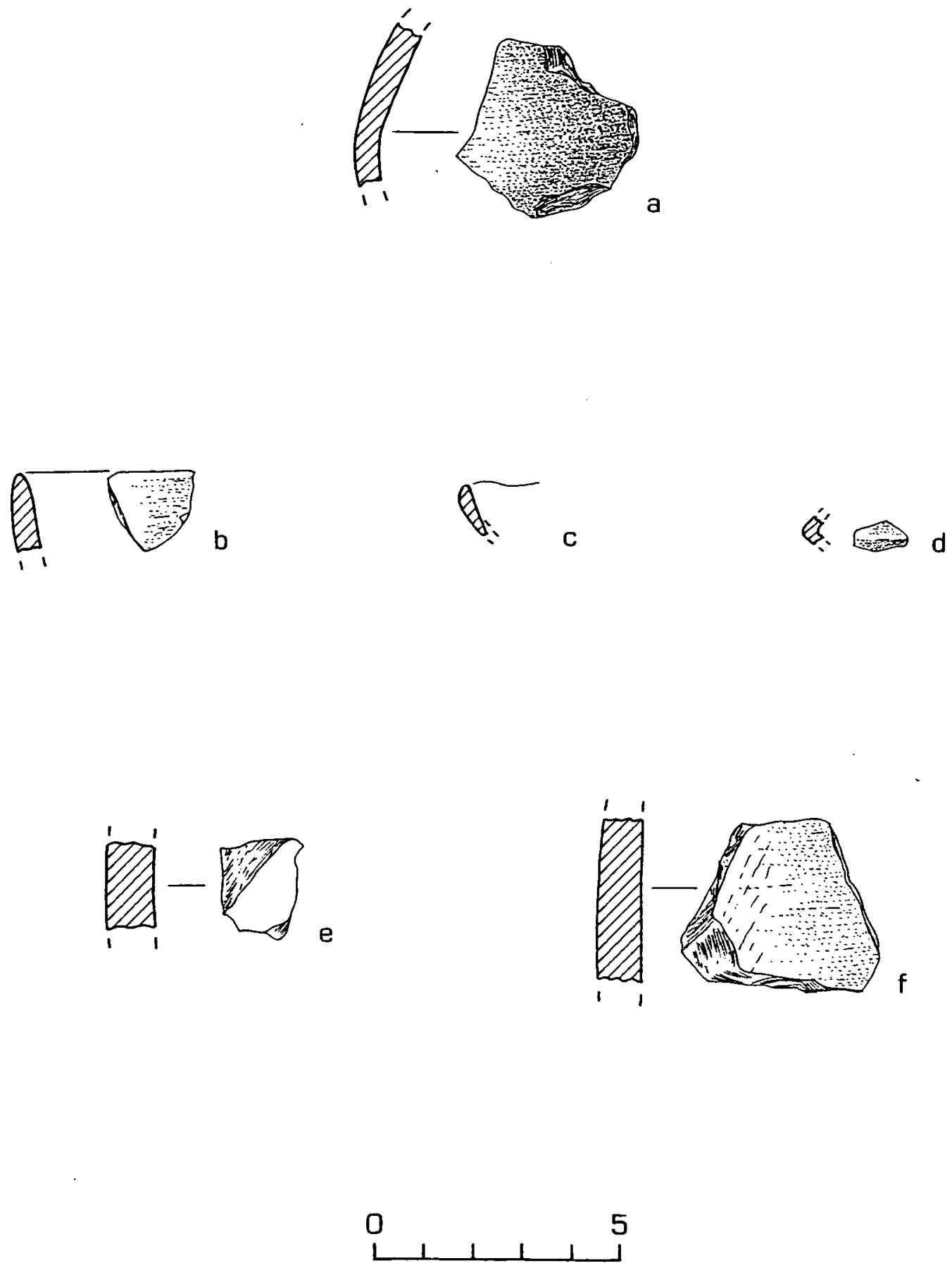

Fig. 3. Cádiz 2001. Fragmentos de cerámica fenicia hallados en la perforación CAD 613 realizada en la Plaza de la Catedral (a-e) y en la CAD 604 realizada en La Viña (f) (según Arteaga et alii 2001). 